\title{
Response Bandwidth Design of Fabry-Perot Sensors for Partial Discharge Detection Based on Frequency Analysis
}

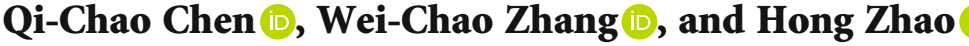 \\ Key Laboratory of Engineering Dielectrics and Its Application, Ministry of Education, School of Electrical and Electronic Engineering, \\ Harbin University of Science and Technology, Harbin 150080, China \\ Correspondence should be addressed to Wei-Chao Zhang; weichaozhang@163.com
}

Received 22 June 2019; Revised 7 September 2019; Accepted 25 October 2019; Published 18 November 2019

Academic Editor: Carlos Ruiz

Copyright (C) 2019 Qi-Chao Chen et al. This is an open access article distributed under the Creative Commons Attribution License, which permits unrestricted use, distribution, and reproduction in any medium, provided the original work is properly cited.

\begin{abstract}
The insulation of power equipment can be effectively assessed by analyzing the acoustic signals originated from partial discharges (PD). Fabry-Perot (F-P) sensors are capable of detecting PD acoustic signals. Although the frequency bandwidth of an F-P sensor is mainly referred to conventional piezoelectric transducer (PZT) sensor, it is still doubtful to identify a suitable bandwidth for fiber sensors in detection of PD signals. To achieve a suitable bandwidth for an F-P sensor, the frequency distribution of PD acoustic emission is investigated, and an extrinsic F-P sensor is designed to detect acoustic signals generated from PD. F-P sensors with different intrinsic frequencies are fabricated as possible design standards of bandwidth for acoustic detection. PD acoustic signals are detected by these F-P sensors and PZT sensors in the experimental system, in which four typical electrode models are employed. The measured results of frequency performance are analyzed in linear and semilogarithmic coordinates. The results show that F-P sensors can effectively detect PD acoustic emissions in both wideband and narrowband modes. Moreover, F-P sensors achieve a higher sensitivity in the narrowband mode. We propose that intrinsic frequency of the F-P sensor should be designed in the frequency range of $100-170 \mathrm{kHz}$ to obtain maximum sensitivity.
\end{abstract}

\section{Introduction}

Partial discharges (PD) can lead to the failure of high-voltage power equipment. The energy released during PD produces effects such as the release of chemical gas and heat, structural changes in materials, and production of ultrasound and electromagnetic signals [1]. Methods for detecting PD include electrical, radio frequency, coupling capacitor, ultraviolet light, acoustic emission, and dissolving gas analysis. The detection of PD by acoustic emission (AE) techniques is immune to electromagnetic interference. In addition, AEbased technologies could provide solutions for locating PD sources. PD detection by AE is based on detecting ultrasonic waves emitted from the PD. Several methods have been studied, and much experience has accumulated. Piezoelectric transducers (PZT) have been the preferred approach to PD detection in transformers. PZT are easy to install and replace; however, PZT performance can be affected by electromagnetic interference. In contrast to PZT, fiber optic sensors have practical advantages: they are immune to electromag- netic interference, can be multiplexed, have high sensitivity, and can be embedded into composite materials [2]. Therefore, fiber optic AE detection technology is also used to detect PD in the insulation of high-voltage power equipment. In the past few years, Mach-Zehnder, Michelson, and Sagnac fiber interferometric technologies have been applied to PD detection [3-5]. In addition, extrinsic Fabry-Perot (F-P) interferometric technology has received attention. F-P interferometric sensors are fabricated as small sensing elements that can simultaneously utilize an F-P cavity with two parallel reflecting surfaces. Many studies have focused on the design and fabrication of F-P sensors due to their compact size and high sensitivity for PD acoustic signals.

Early extrinsic F-P sensors for PD detection are presented by Deng [6]. The interferometric structure is produced between the end face of a fiber and a mirrored quartz membrane. Microelectromechanical system (MEMS) technology is used to fabricate an optical extrinsic F-P interferometric sensor $[7,8]$. An F-P sensor with high thermal stability is reported by Akkaya [9], in which a novel high-yield 


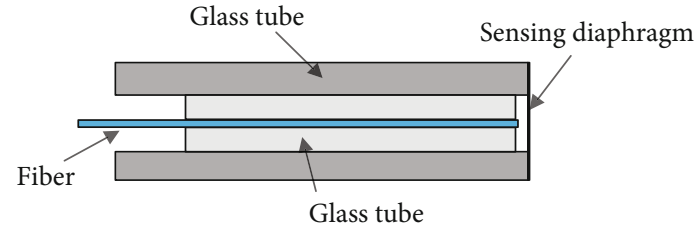

Figure 1: Schematic structure of an extrinsic F-P interferometer sensor.

silicate-bonding process and a packaging technology are established to fabricate acoustic fiber sensors. A fringe visibility-enhanced F-P sensor has been proposed, and a high signal-to-noise ratio (SNR) of $42.92 \mathrm{~dB}$ in $300 \mathrm{kHz}$ was achieved [10]. Interferometric optical fiber sensors with high stability and low harmonic distortion have been developed with a single-path differential-divide phase generation carrier demodulation algorithm to achieve high sensitivity, good linearity, and a large dynamic range [11].

Although many reports of F-P sensor design, fabrication, and characterization have been published, the relationship between the frequency response characteristics of F-P sensors and PD AE signals has rarely been studied. There is no universal standard for the response frequency of a $\mathrm{PD}$ acoustic detector. IEEE Std C57.127 demonstrates that acoustics generated from PD have a wideband frequency from $20 \mathrm{kHz}$ to $500 \mathrm{kHz}$ [12]. Most PZT sensors used for PD detection have a resonant response at $150 \mathrm{kHz}$. Studies have shown that the response frequency of an acoustic PD sensor should be in the range of $20-200 \mathrm{kHz}$ [13] and that the response frequency of a sensor should be less than $200 \mathrm{kHz}$ for PD acoustic detection [14]. However, some have proposed that the sensor bandwidth should be between $100 \mathrm{kHz}$ and $300 \mathrm{kHz}$ [15-17]. Due to the randomness of $\mathrm{PD}$ signals, the relationship between the response frequency of sensors and PD acoustic signals is worth considering.

In the present work, F-P sensors with different parameters and response frequencies are designed to detect $\mathrm{PD} \mathrm{AE}$ signals in a liquid dielectric. In the experimental system, F$\mathrm{P}$ sensors and PZT sensors are used together to detect PD AE. The frequency spectrum of different F-P sensors and PZT sensors are compared, and the frequency distribution of PD AE is analyzed and demonstrated. According to the results from four F-P sensors, we propose a frequency design and method for using F-P sensors for PD detection.

\section{Materials and Methods}

2.1. PD Sensor Design and Manufacture. A sectional view of the design of an extrinsic fiber F-P sensor is illustrated in Figure 1. The extrinsic F-P sensor we designed consists of two glass tubes with different diameters, a pigtail of a single-mode fiber, and a sensing diaphragm made of silica glass. The sensing diaphragm has a reflectance coating of $50 \%$ reflectivity.

When acoustic waves generated by a PD impinge on the diaphragm of an F-P sensor to induce deflection, the cavity length is modulated by the deformation of the diaphragm. The most important characteristics of an F-P sensor are

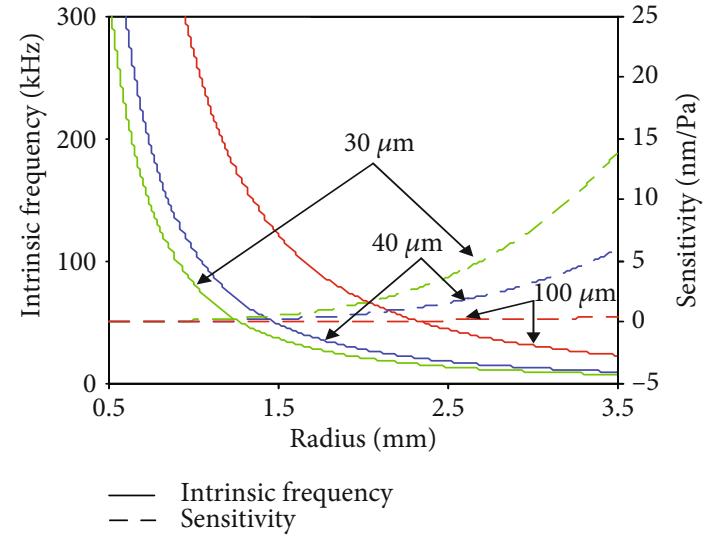

FIgURE 2: Intrinsic frequencies and sensitivity of circular diaphragms with different thicknesses.

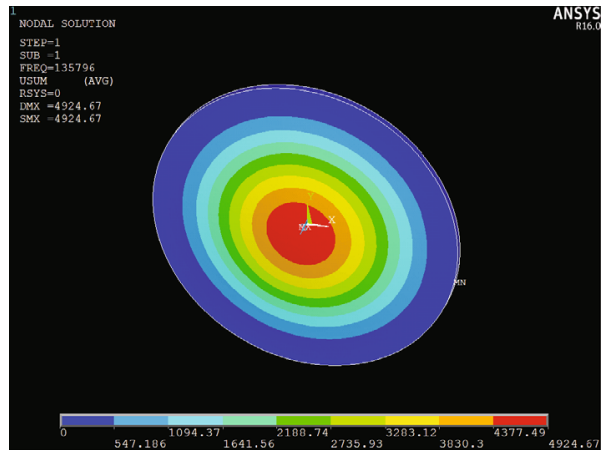

FIgURE 3: Spatial distribution of response frequency in an F-P sensor by ANSYS simulation.

TABLE 1: Intrinsic frequencies of F-P sensors with different physical dimensions.

\begin{tabular}{lccc}
\hline Sensor & $\begin{array}{c}\text { Diameter } \\
(\mathrm{mm})\end{array}$ & $\begin{array}{c}\text { Thickness } \\
(\mu \mathrm{m})\end{array}$ & $\begin{array}{c}\text { Simulation intrinsic } \\
\text { frequency }(\mathrm{kHz})\end{array}$ \\
\hline F-P \#1 & 1.8 & 50 & 169.7 \\
F-P \#2 & 1.8 & 40 & 135.8 \\
F-P \#3 & 1.1 & 20 & 181.9 \\
F-P \#4 & 1.5 & 40 & 195.3 \\
\hline
\end{tabular}

center deflection and intrinsic frequency. Center deflection affects the sensitivity of an F-P sensor, and intrinsic frequency determines whether the sensor operates in a narrowband or wideband mode. Based on the theory of elasticity, the intrinsic frequency and diaphragm sensitivity vary with diaphragm radius, which is shown for diaphragms having radii of 30, 40, and $100 \mu \mathrm{m}$ in Figure 2. The intrinsic frequency of a diaphragm decreases as the radius increases. The relationship between intrinsic frequency and diaphragm radius is the inverse of that between sensitivity and radius.

The acoustic signals from partial discharges are in a region ranging from approximately 20 to $500 \mathrm{kHz}$. It is difficult to determine the most suitable frequency for $\mathrm{PD}$ 

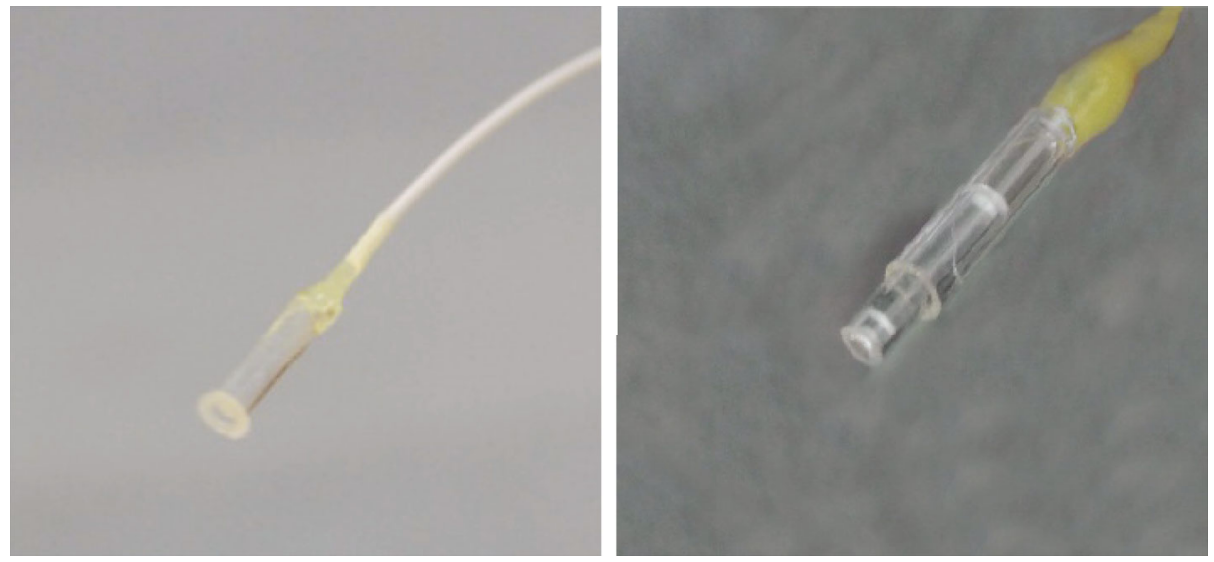

Figure 4: Images of F-P sensor samples.

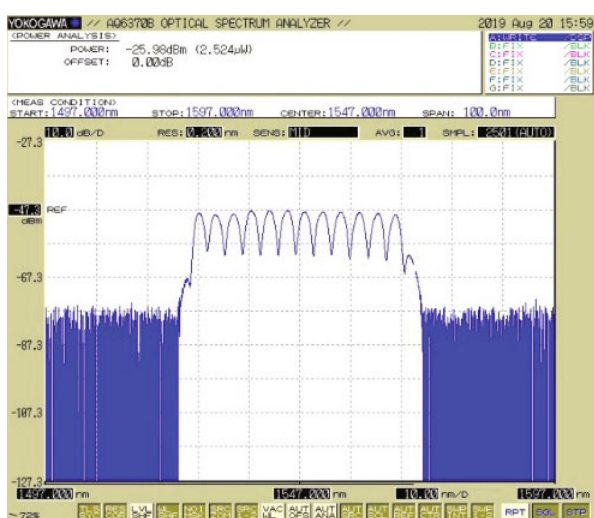

(a)

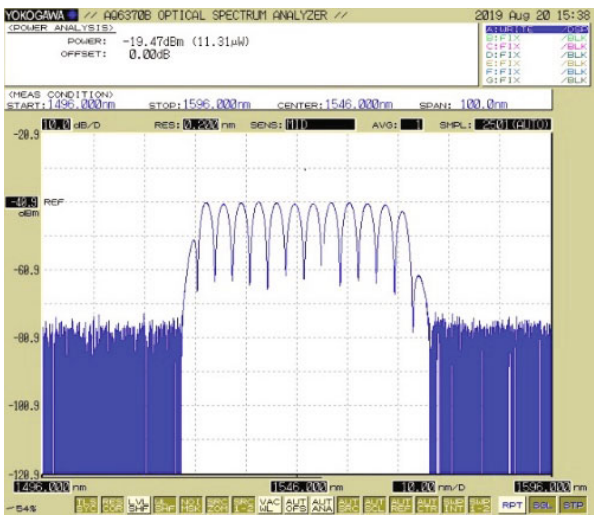

(c)

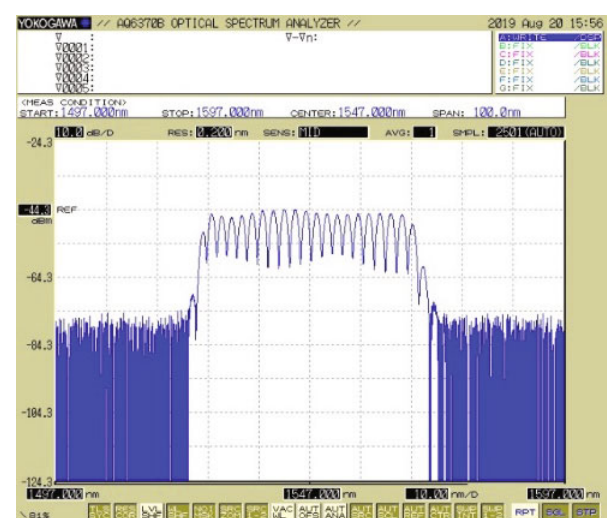

(b)

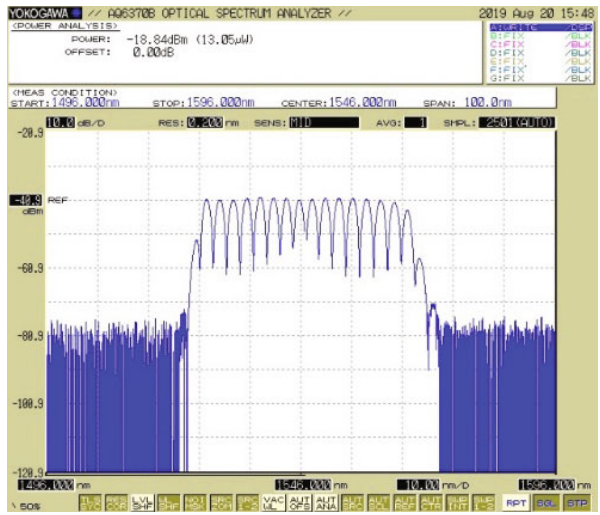

(d)

Figure 5: Spectra of F-P sensors: (a) F-P sensor \#1, (b) F-P sensor \#2, (c) F-P sensor \#3, and (d) F-P sensor \#4.

detection. To study the relationship between the effectiveness of PD and the frequency response characteristics of a sensor, we individually manufactured several F-P sensors with intrinsic frequencies in different wavebands. Based on the theory of elasticity, the intrinsic frequency of an F-P sensor is calculated using the finite element method (FEM). From the ANSYS simulation shown in Figure 3, the intrinsic frequency of the designed F-P sensor (thickness $=40 \mu \mathrm{m}$, diameter $=1.8 \mathrm{~mm}$ ) approaches $135.8 \mathrm{kHz}$. In comparison, three other sensors were designed by changing dimensions and were simulated to estimate their intrinsic frequency characteristics; the results are listed in Table 1.

Sensors with different response frequencies were made by bonding fibers, silica ferrules, silica glass tubes, and diaphragms in the laboratory. Images of the detecting heads of representative sensors are shown in Figure 4, and the tested interference spectrum of sample F-P sensors is shown in Figure 5. 


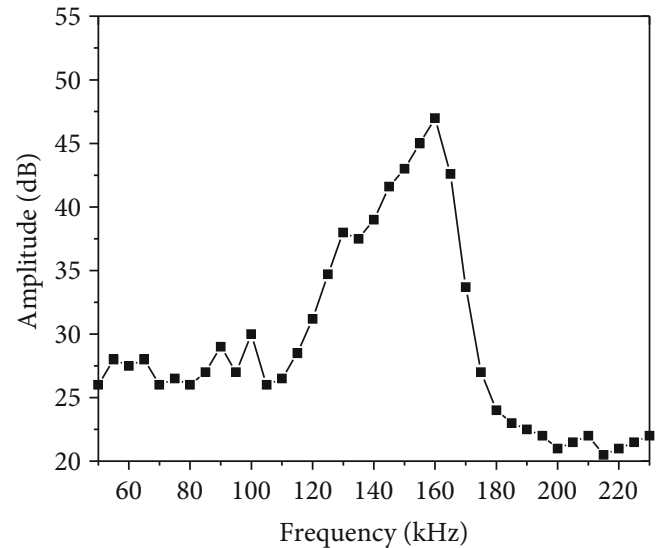

(a)

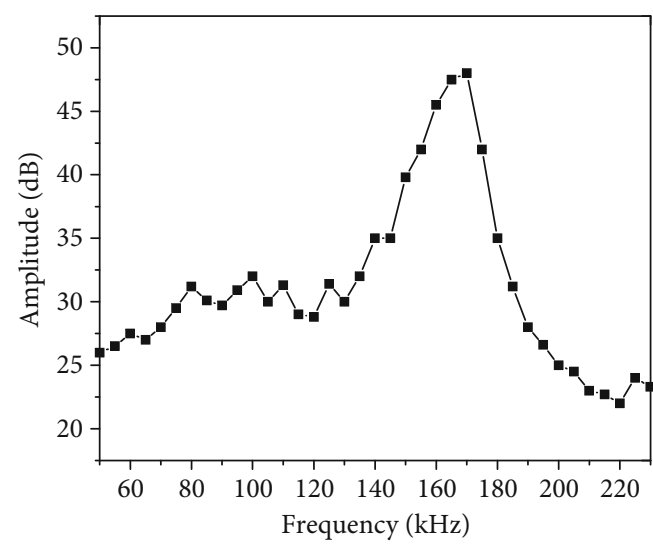

(c)

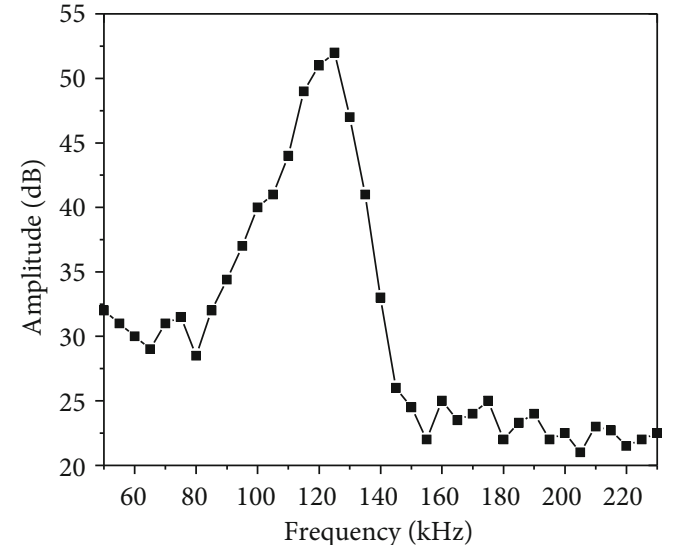

(b)

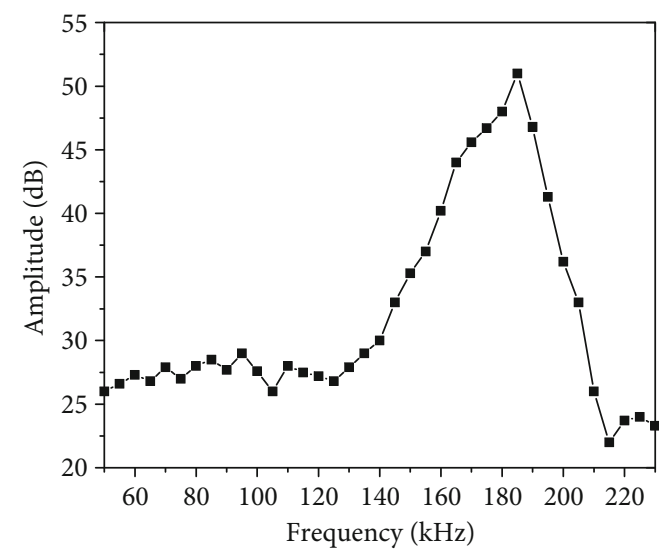

(d)

Figure 6: Frequency response curves of F-P sensors: (a) F-P sensor \#1, (b) F-P sensor \#2, (c) F-P sensor \#3, and (d) F-P sensor \#4.

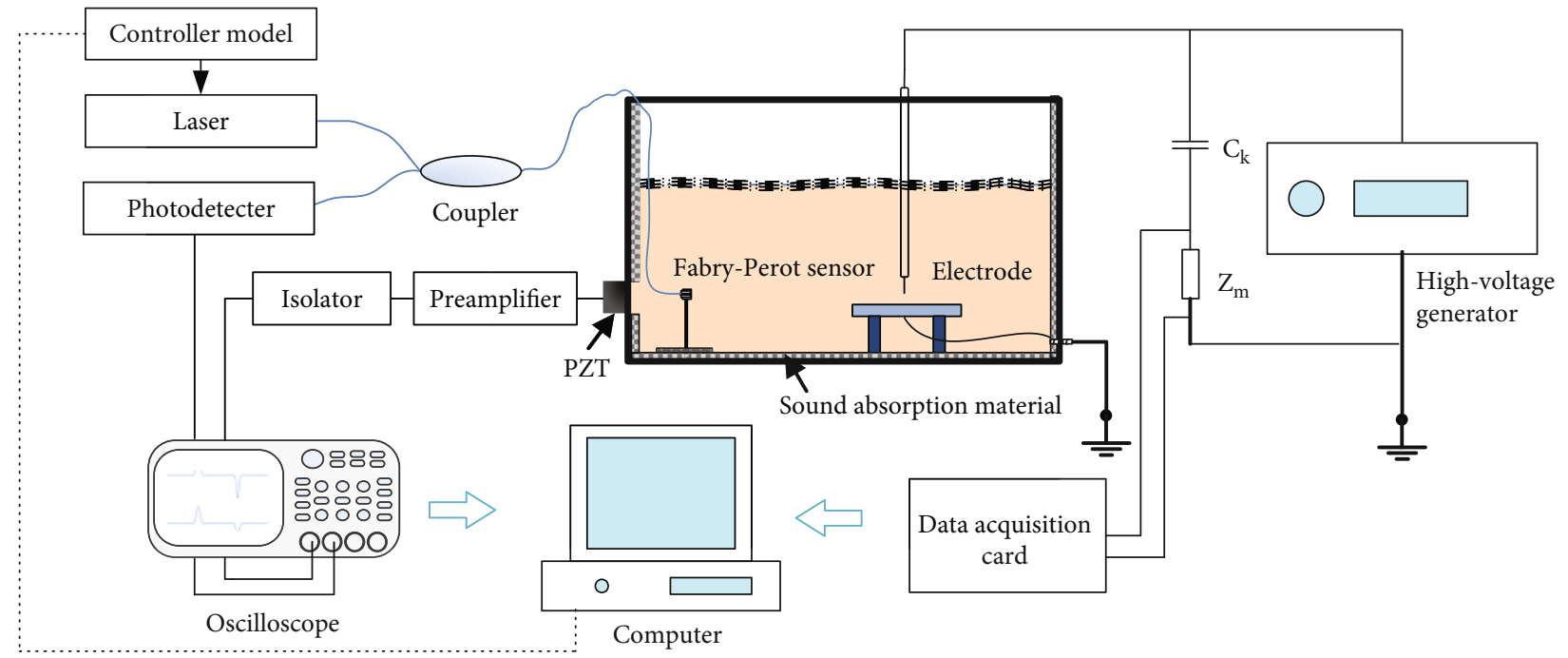

Figure 7: Schematic of the PD detection system.

2.2. Frequency Response Tests. The F-P sensors were designed primarily based on diaphragm vibration to detect PD acoustic signals. When the frequency of the acoustic emission is higher than the intrinsic frequency of an F-P sensor, the F-P diaphragm will exhibit high attenuation. If the frequency of the acoustic emission $(f)$ is higher than the intrinsic frequency of the F-P sensor $\left(f_{0}\right)$, the amplitude of the F-P diaphragm will decrease to almost zero. However, 


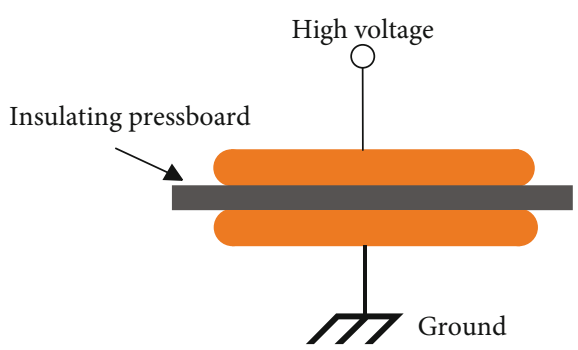

(a)

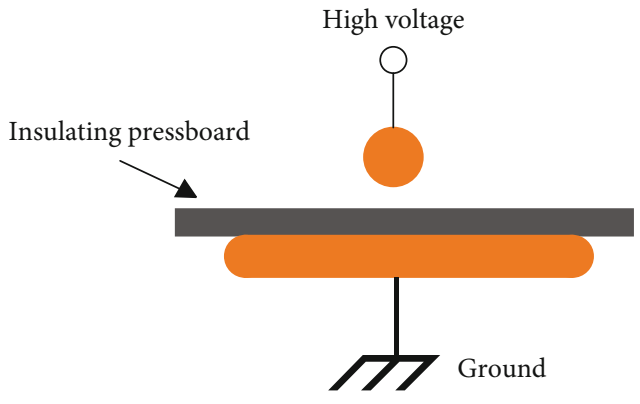

(c)

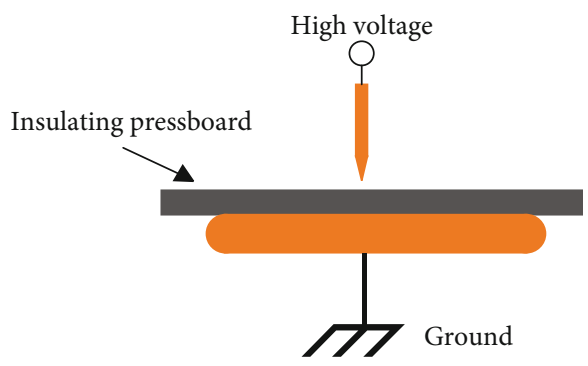

(b)

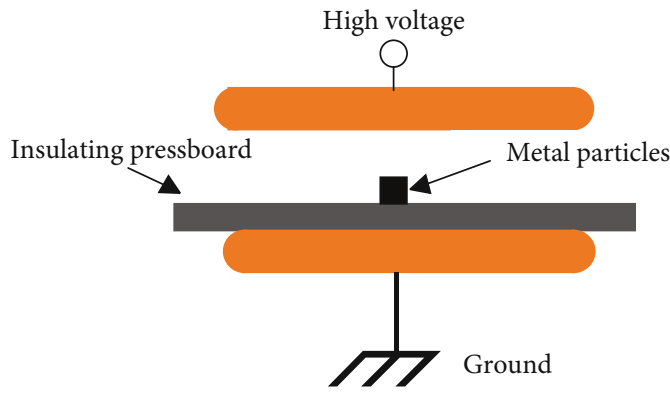

(d)

Figure 8: Electrode models used to generate PD: (a) plate-plate model, (b) needle-plate model, (c) ball-plate model, and (d) floating model.

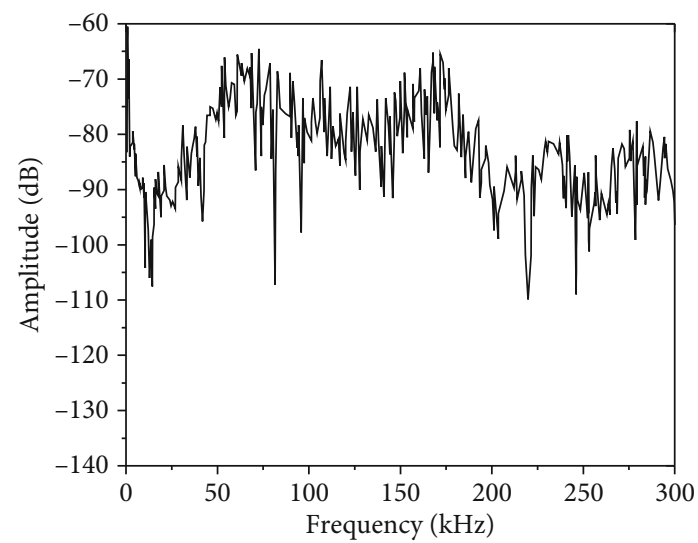

FIgURE 9: Amplitude-frequency characteristic curve of the PZT transducer.

if $f$ equals $f_{0}$, the F-P sensor will remain in a resonance state, which generates a high response region near $f_{0}$; if $f$ is lower than $f_{0}$, the amplitude persists at a constant value that is smaller than the resonance response.

To investigate the amplitude-frequency response of F-P sensors, ultrasonic waves are generated by a piezoelectric sensor, in which the frequency is changed continuously from 50 to $230 \mathrm{kHz}$. F-P sensors (\#1, \#2, \#3, and \#4) are used to measure the acoustic signals. The frequency response curves for each F-P sensor are shown in Figures 6(a)-6(d), respectively. According to the test results, F-P sensor \#1 has a high response region ranging from approximately 140 to $170 \mathrm{kHz}$, as presented in Figure 6(a). Similarly, the high response region frequencies are $100-135 \mathrm{kHz}, 140-175 \mathrm{kHz}$, and
$170-200 \mathrm{kHz}$ for F-P sensors \#2, \#3, and \#4, respectively, as illustrated in Figures 6(b)-6(d).

\section{Results and Discussion}

3.1. PD Detection Experimental System. The experimental system was established to detect $\mathrm{AE}$ from $\mathrm{PD}$ in liquid insulation. As shown in Figure 7, a tank $(500 \times 500 \times 1000 \mathrm{~mm})$ was filled with insulating oil. A partial discharge generator model connected to a high-voltage transformer was immersed in the insulating oil. F-P sensors were immersed in the oil to couple AE signals from the PD source. A demodulation device was designed based on the vibration intensity. A $1550 \mathrm{~nm}$ wavelength DFB laser was used as the source in the demodulation system. As shown in Figure 6, the light beam of the DFB laser injects into the fiber and transmits to the F-P sensor head through a coupler. The light beam is partially reflected at the end of fiber tip. The remaining light emits from the fiber tip, propagates to the silica diaphragm, and is reflected by the fiber tip, resulting in interference in the fiber tip. Half of the interference light that is returned is absorbed into the coupler and detected by the photodetector. The output voltage of the photodetector is transformed into a digital signal by an A/D converter, and a temperature controller is used to stabilize the operation point of the detection system. The wavelength of the DFB laser is tunable by the heat sink, which is controlled by the temperature controller. When the operation point of the F-P sensor changes, the temperature controller will tune the center wavelength of the laser to stabilize the operation point.

In the experimental system, four typical PD electrode models are designed to generate PD acoustic signals. Plateplate, needle-plate, ball-plate, and floating electrode models 


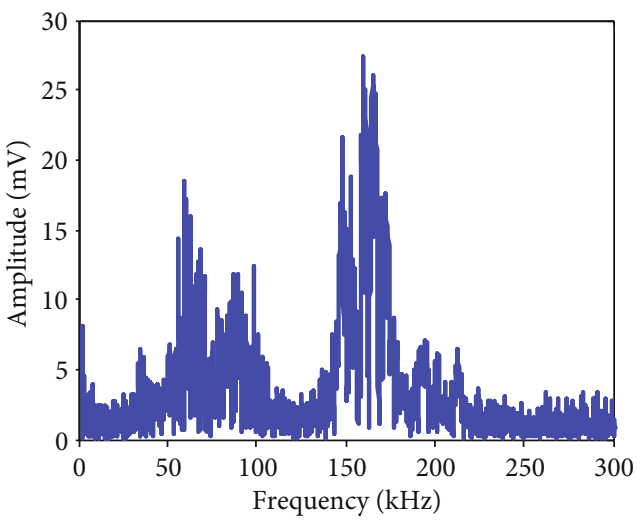

(a)

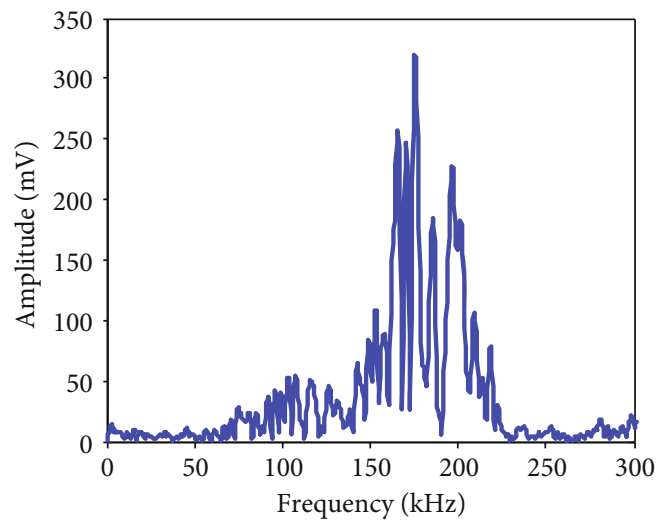

(c)

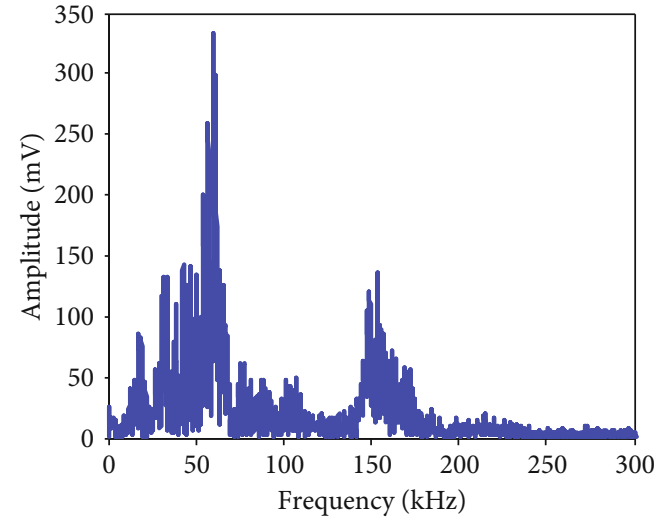

(b)

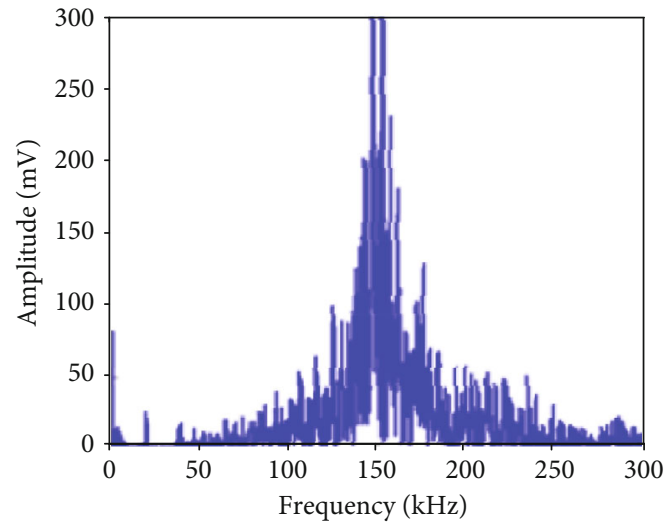

(d)

Figure 10: Amplitude-frequency curves of the PZT transducer: (a) plate-plate electrode model, (b) ball-plate electrode model, (c) needle-plate electrode model, and (d) floating electrode model.

are presented in Figure 8. An insulating pressboard with a thickness of $1 \mathrm{~mm}$ is inserted between electrodes. To analyze the frequency distribution characteristics of PD acoustic signals, a piezoelectric (PZT) sensor (SR15, Soundwel Technology, Ltd., China) mounted externally on the oil tank is used to synchronously detect acoustic signals from PD with the FP sensor. The amplitude-frequency characteristic curve of the PZT sensor is also recorded for comparison, as shown in Figure 9.

3.2. Frequency Analysis of $P D A E$ Generated by Different Electrode Models. According to the IEEE Std C57.127 [12], the frequency range of PD AE is $20-500 \mathrm{kHz}$. Typically, a PZT sensor is designed as a narrowband sensor to detect PD AE. Due to the high attenuation of diaphragm vibration when the acoustic frequency is higher than the intrinsic frequency of an F-P sensor, F-P sensors are typically designed as wideband sensors. However, no frequency criterion in the design of PD acoustic sensors has been established to date. In these experiments, the frequency characteristics of typical models are analyzed using the manufactured F-P sensors and a PZT sensor.

\subsubsection{Frequency Distribution Characteristics of Detection} with the PZT Sensor. The PZT sensor is used to measure $\mathrm{PD}$ acoustic signals from the four electrode models described above. The frequency curves shown in Figure 10 were calculated by the Fourier transform method. The peak frequency values of PD acoustic signals generated from the plate-plate electrode model are primarily at 60 and $160 \mathrm{kHz}$, as shown in Figure 10(a). The frequency distribution of the ball-plate electrode model is similar to the plate-plate electrode model (Figure 10(b)). A small response value appears at $100 \mathrm{kHz}$ in the frequency spectra of both the plate-plate and ballplate electrode models. However, for needle-plate and floating electrode models, the peak values of PD acoustic signals occur at $160 \mathrm{kHz}$ and $150 \mathrm{kHz}$, respectively, as shown in Figures 10(c) and 10(d). The PZT testing results show that PD acoustic signals have high amplitudes in the frequency ranges of $50-70 \mathrm{kHz}$ and $150-160 \mathrm{kHz}$. To avoid environmental noise, response outputs are usually ignored near $50 \mathrm{kHz}$. Therefore, it is difficult to distinguish between the four typical electrode models based on PD acoustic signals detected by PZT, because peak frequency values occur at $150-160 \mathrm{kHz}$ for all models; similar acoustic frequency distributions are seen between plate-plate and ball-plate electrodes, and analogous acoustic signals are seen between needle-plate and floating electrodes.

3.2.2. Frequency Distribution Characteristics of Detection with Fabry-Perot Sensors. In the detection results with the PZT sensor, PD acoustics generated from the ball-plate and 


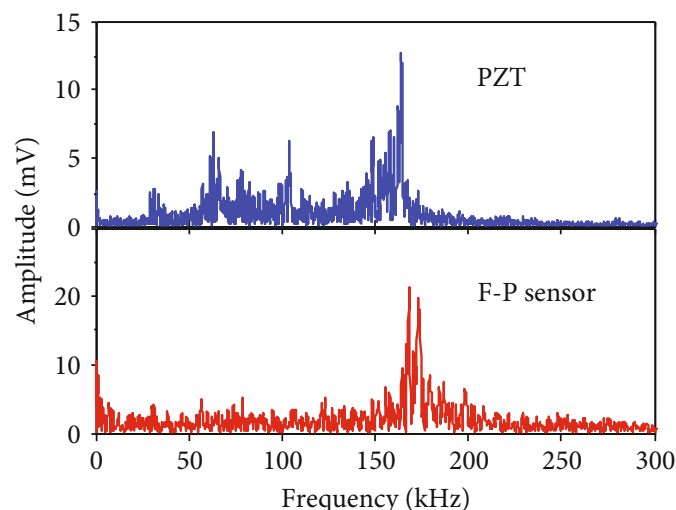

(a)

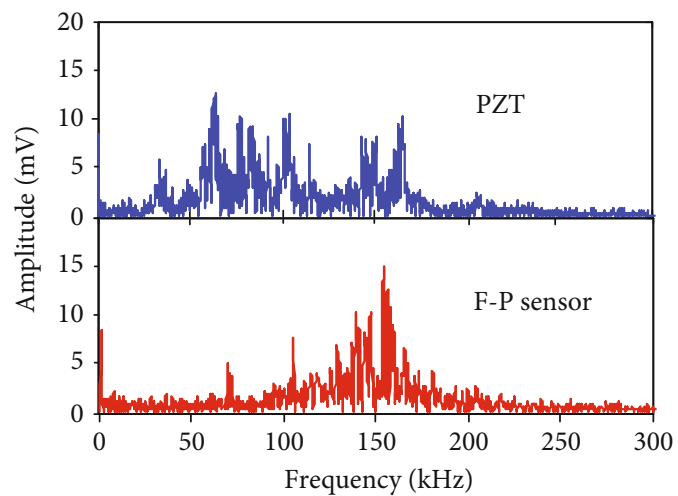

(c)

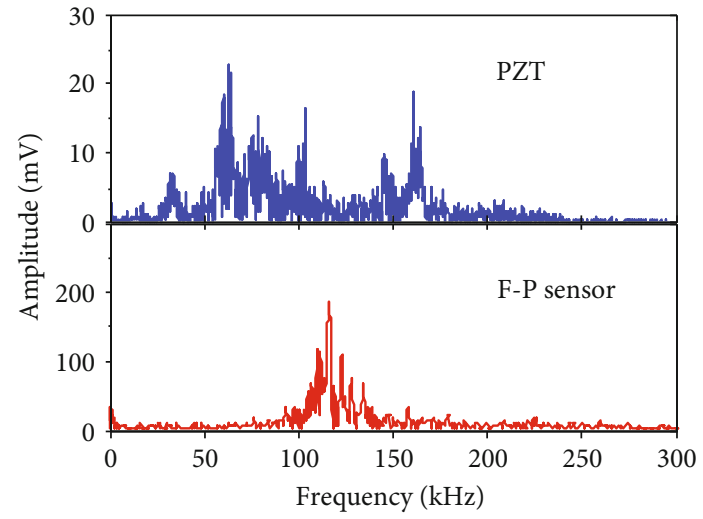

(b)

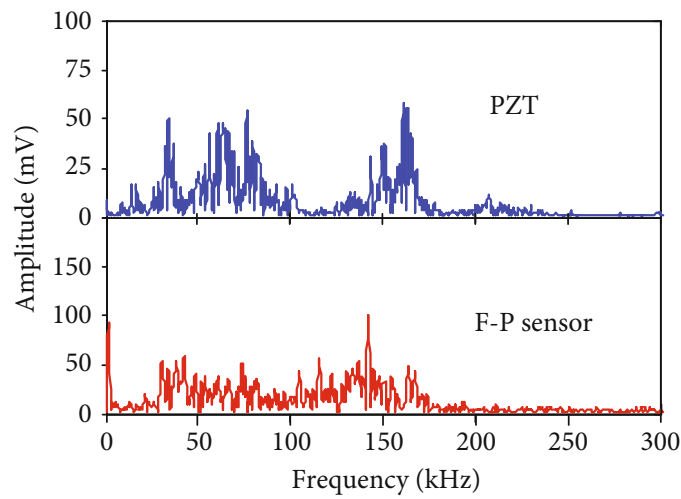

(d)

FIgURE 11: Frequency spectra in linear coordinates for PD detection with the ball-plate electrode model: (a) F-P sensor \#1, (b) F-P sensor \#2, (c) F-P sensor \#3, and (d) F-P sensor \#4.

plate-plate electrode models have similar frequency distribution characteristics. Therefore, the ball-plate, needle-plate, and floating electrode models were chosen to generate PD acoustic signals.

The manufactured F-P sensors were used to detect acoustic signals generated by the ball-plate electrode model. Figures 11(a)-11(d) show the amplitude-frequency curves of acoustic signals detected by the four F-P sensors and the PZT sensor plotted in a linear coordinate system. The peak frequency values of F-P sensors appear near $160 \mathrm{kHz}$, $120 \mathrm{kHz}, 170 \mathrm{kHz}$, and $150 \mathrm{kHz}$, respectively. Compared to the intrinsic frequencies of these F-P sensors, peak values of the PD acoustic signal frequency spectra appear near the intrinsic frequencies of the F-P sensors, except in the case of F-P sensor \#4. That is, F-P sensors \#1, \#2, and \#3 are working in the resonance mode based on the simulated PD acoustics. PD acoustic signals contain the frequencies of $160 \mathrm{kHz}$, $120 \mathrm{kHz}$, and $170 \mathrm{kHz}$. However, the intrinsic frequency of F-P sensor \#4 is approximately $190 \mathrm{kHz}$, but the peak frequency value with F-P sensor \#4 appears near $150 \mathrm{kHz}$ when detecting $\mathrm{PD}$ signals from the ball-plate electrode model. F-P sensor \#4 is working out of resonance mode. It is concluded that PD AE energy from the ball-plate electrode model is reduced near the frequency of $190 \mathrm{kHz}$.

When plotted using linear coordinates, peak frequency values could be easily acquired from amplitude-frequency curves, but the frequency distribution ranges of $\mathrm{PD} A \mathrm{AE}$ were ambiguous. Using logarithmic coordinates can help visualize the frequency distribution range of acoustic signals. Therefore, the amplitude-frequency curves above were redrawn using semilogarithmic coordinates. Amplitude-frequency spectra for semilogarithmic coordinates were calculated from Figure 11 and are presented in Figure 12.

Comparing the curves of the PZT sensors, their amplitudes versus frequency distributions are similar. All signals measured by PZT appear as higher gain responses in the frequency ranges of $50-70 \mathrm{kHz}$ and $150-170 \mathrm{kHz}$, which are similar to the higher gain response ranges for the PZT characteristic curve presented in Figure 9. The amplitudes seen in the frequency ranges of $50-70 \mathrm{kHz}$ and $150-170 \mathrm{kHz}$ are approximately $10 \mathrm{~dB}$ higher than those in the frequency range of $80-140 \mathrm{kHz}$. The amplitude-frequency characteristic curve of PZT for PD detection presents the same distribution pattern, as shown in Figures $12(\mathrm{a})-12(\mathrm{~d})$, indicating that the excitation signal of the PZT sensor is a broadband signal with a frequency range of $0-200 \mathrm{kHz}$. Finally, it is concluded that the PD acoustic signals from the ball-plate model are continuous and adequate in the frequency range of $<200 \mathrm{kHz}$.

The amplitude-frequency curves for PD detection with the F-P sensors have different characteristics. The amplitude-frequency curve of F-P sensor \#1 has higher response values in the frequency range of $150-170 \mathrm{kHz}$, as shown in Figure 12(a), which is similar to its resonance 


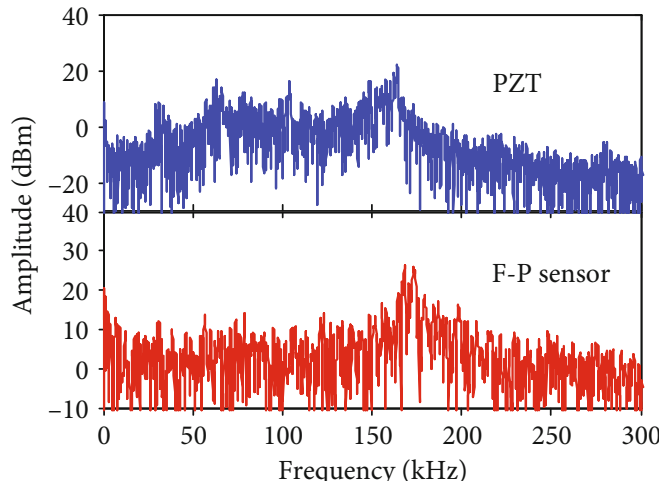

(a)

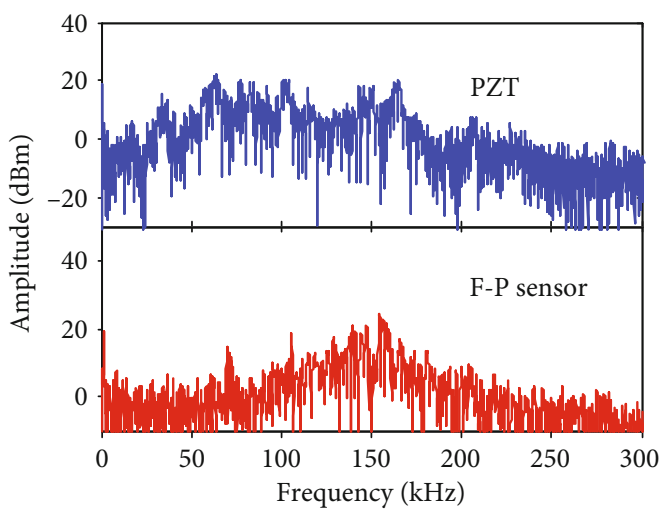

(c)

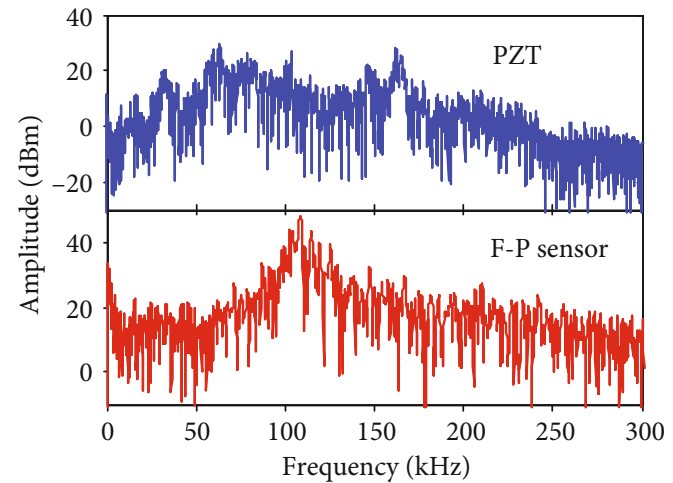

(b)

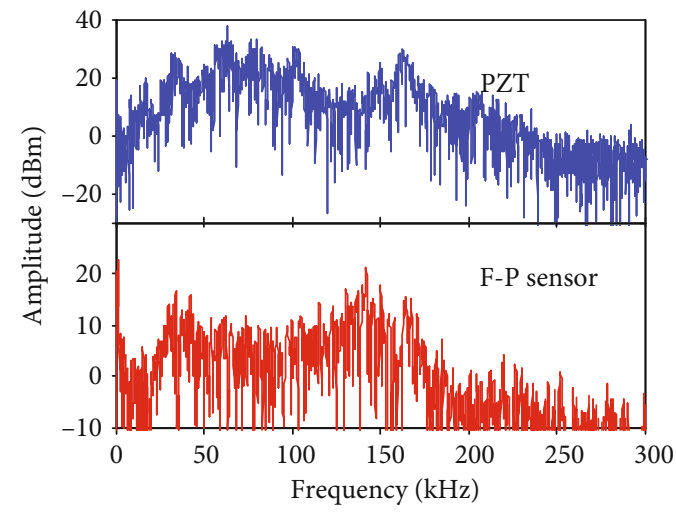

(d)

FIGURE 12: Frequency spectra in logarithmic coordinates for PD detection with the ball-plate electrode model: (a) F-P sensor \#1, (b) F-P sensor \#2, (c) F-P sensor \#3, and (d) F-P sensor \#4.

curve in that resonance oscillation takes place when F-P sensor \#1 is excited by PD acoustic emission. The results show that the PD acoustic signal frequency includes the intrinsic frequency of F-P sensor \#1. Meanwhile, F-P sensors $\# 2$ and \#3 present similar response characteristics as those of F-P sensor \#1, as shown in Figures 12(b) and 12 (c). F-P sensor \#2 has higher response values in the frequency range of $100-130 \mathrm{kHz}$, which is the same as its resonance curve. And F-P sensor \#3 also has higher response values near its intrinsic frequency, which is in the frequency range of 130 $-170 \mathrm{kHz}$. Both F-P sensor \#2 and F-P sensor \#3 work in resonance mode when they detect $\mathrm{PD}$ acoustics. It is concluded that PD acoustic signals include the intrinsic frequencies of F-P sensors \#2 and \#3, which are in the ranges of $100-130 \mathrm{kHz}$ and $130-170 \mathrm{kHz}$. According to the PD detection amplitude-frequency characteristics of F-P sensors $\# 1$, \#2, and \#3, it is shown that PD acoustic emissions generated from ball-plate electrode model have high amplitudes in the $100 \mathrm{kHz}$ to $170 \mathrm{kHz}$ frequency range.

When F-P sensors \#1, \#2, and \#3 detect PD AE signals, resonance oscillation occurs, i.e., when $\mathrm{PD}$ acoustic signals contain the intrinsic frequency of an F-P sensor, that sensor will operate in resonance mode, as F-P sensors \#1, \#2, and \#3 do; therefore, these F-P sensors are working in the narrowband mode for PD detection. However, F-P sensor \#4 displays different results. The amplitude-frequency curve of F-P sensor \#4 has a flat amplitude response in the frequency
TABLE 2: Frequency distribution characteristics of sensors for PD detection with the ball-plate electrode model.

\begin{tabular}{lccc}
\hline No. & $\begin{array}{c}\text { Simulation } \\
\text { intrinsic } \\
\text { frequency } \\
(\mathrm{kHz})\end{array}$ & $\begin{array}{c}\text { High response } \\
\text { region ranges of F-P } \\
\text { sensors tested }(\mathrm{kHz})\end{array}$ & $\begin{array}{c}\text { Response frequency } \\
\text { distribution for PD } \\
\text { detection }(\mathrm{kHz})\end{array}$ \\
\hline $\begin{array}{l}\text { F-P } \\
\text { sensor } \\
\# 1\end{array}$ & 163 & $140-170$ & $150-170$ \\
$\begin{array}{l}\text { F-P } \\
\text { sensor }\end{array}$ & 129 & $100-135$ & $100-130$ \\
$\# 2$ & & & $145-170$ \\
$\begin{array}{l}\text { F-P } \\
\text { sensor }\end{array}$ & 169 & $145-175$ & \\
$\# 3$ & & & $40-170$ \\
F-P & & & \\
sensor & 195 & $170-200$ & \\
$\# 4$ & & & \\
\hline
\end{tabular}

range of $40-170 \mathrm{kHz}$. While in the intrinsic frequency range of $180-200 \mathrm{kHz}, \mathrm{F}-\mathrm{P}$ sensor \#4 has smaller amplitude values. Results with F-P sensor \#4 show that PD acoustic signals have less energy in the frequency range of $180-200 \mathrm{kHz}$. Meanwhile, it is defined that the F-P sensor is working in the wideband mode for PD detection. 


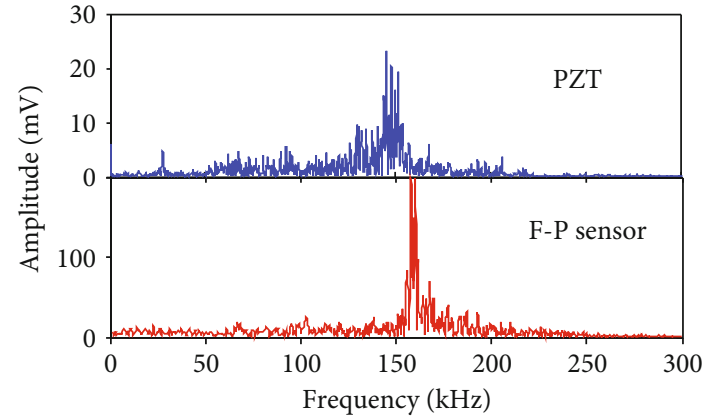

(a)

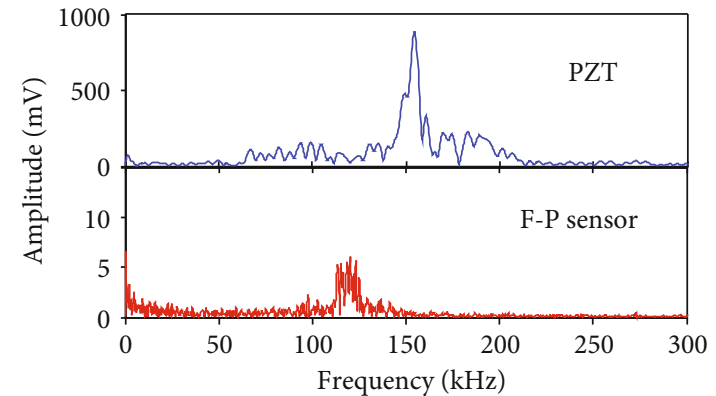

(b)

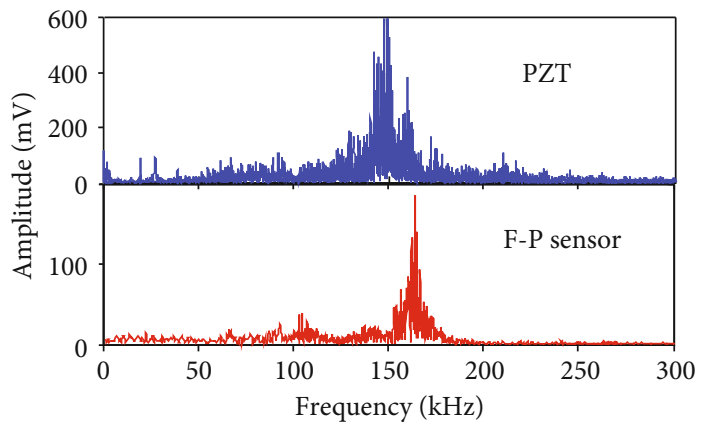

(c)

FIGURE 13: Frequency spectra in logarithmic coordinates for PD detection with the needle-plate electrode model: (a) F-P sensor \#1, (b) F-P sensor \#2, and (c) F-P sensor \#3.

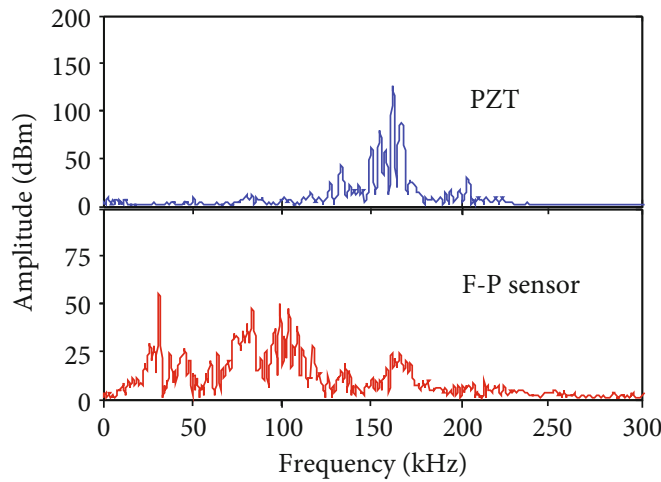

(a)

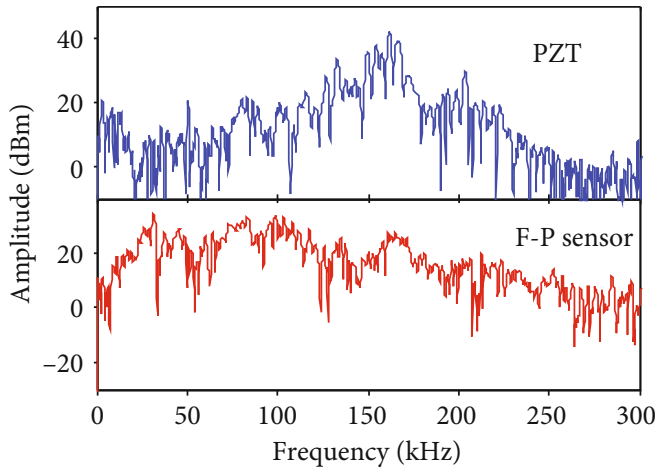

(b)

FIgURE 14: Amplitude-frequency curves of PD detection with F-P sensor \#4 and the needle-plate electrode model: (a) logarithmic coordinates and (b) semilogarithmic coordinates.

Based on the above analysis, the high response region ranges tested and response frequency distribution for PD detection are presented in Table 2. Response frequency distributions are located in the high response region range when F-P sensors \# 1 , \#2, and \#3 are used to detect PD acoustics. The three F-P sensors work in the resonance mode (narrowband mode). However, the response frequency distribution for PD detection and the high response region ranges of F-P sensor \#4 are mismatched. F-P sensor \#4 operates in the wideband mode.

In the needle-plate electrode experiment system, F-P sensors \#1, \#2, and \#3 still perform with narrowband properties. Resonance oscillation occurs when the three sensors detect PD acoustics. The higher response frequencies are similar to the results with the ball-plate electrode model. The amplitude-frequency curves of F-P sensors \#1, \#2, and \#3 are shown in Figure 13.

Compared with F-P sensors \#1, \#2, and \#3, F-P sensor \#4 also presents different properties with the needle-plate electrode model, which works in the wideband mode as it did with the ball-plate electrode model. Figure 14(a) shows signals detected by F-P sensor \#4 in linear coordinates. Peak frequency values appear near $40,80,100$, and $165 \mathrm{kHz}$, respectively. PD detection with F-P sensor \#4 occurs over a wide frequency range, but the response value is smaller near the intrinsic frequency of F-P sensor \#4. Meanwhile, as 


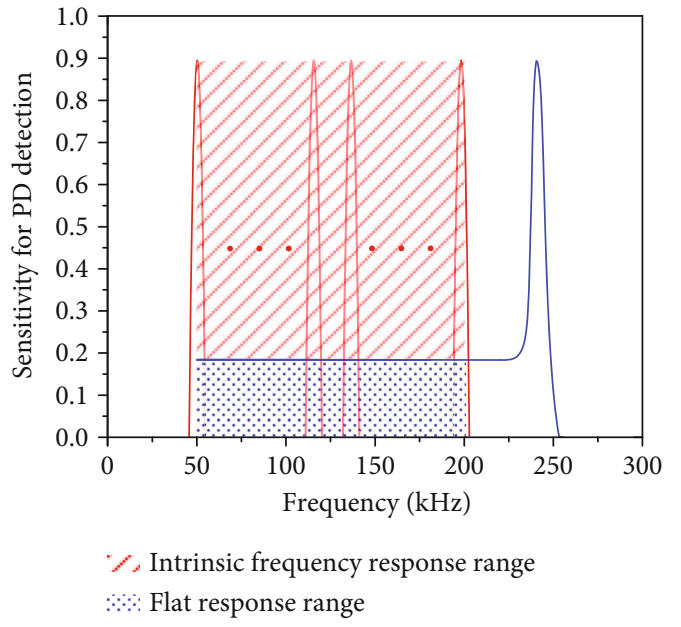

Figure 15: Detection sensitivity for PD detection versus the intrinsic frequency of an F-P sensor.

shown in Figure 14(b), the amplitude-frequency curve in semilogarithmic coordinates is almost flat in the 40$180 \mathrm{kHz}$ frequency range. The results demonstrate that acoustic signals are primarily in the frequency range of 40$80 \mathrm{kHz}$, and the PD acoustic frequency is distributed farther from the intrinsic frequency of F-P sensor \#4 with the needle-plate electrode model. Lastly, all the manufactured F-P sensors and the PZT sensor are used to detect PD acoustic signals with the floating electrode system. Because the floating metal particle in the oil insulation is analogous to a needle structure, PD acoustics generated by the floating electrode model were similar to those seen with the needle-plate electrode model.

In conclusion, the intrinsic frequency of a sensor affects the sensitivity of $\mathrm{PD} \mathrm{AE}$ detection. When the intrinsic frequency is designed in the frequency of PD acoustic signals, F-P sensors will work in the intrinsic frequency response range (red region in Figure 15). When the intrinsic frequency of a sensor is higher than the frequency of PD acoustic signals, F-P sensors will work in the flat response range (blue region in Figure 15). In general, the response amplitude while working in the intrinsic frequency response range is much higher than that in the flat response range. The F-P sensor sensitivity for PD detection is contrasted in different response ranges, as shown in Figure 15.

\section{Conclusions}

In this study, we developed an extrinsic fiber Fabry-Perot sensor for PD AE detection. During the detection of PD with needle-plate, plate-plate, ball-plate, and floating electrode models, the acoustic frequency distribution of $\mathrm{PD}$ was analyzed. The experimental results show that PD AE has high amplitudes in the frequency range of $50-170 \mathrm{kHz}$ with each of the four electrode models. F-P sensors detected PD AE regardless of whether its intrinsic frequency was in the $\mathrm{PD}$ $\mathrm{AE}$ frequency range or higher than the $\mathrm{PD} A \mathrm{AE}$ frequency range. An F-P sensor operates effectively in resonant narrowband mode when its intrinsic frequency is in the PD AE frequency range. Nevertheless, when the intrinsic frequency of an F-P sensor is higher than the PD AE frequency range, it operates effectively in wideband mode for PD detection.

According to the experimental results, two principles for designing F-P sensors for PD AE detection are presented: (i) The intrinsic frequency the F-P sensor can be higher than $200 \mathrm{kHz}$, and a flat response in the frequency range of 50$200 \mathrm{kHz}$ should be used to detect signals. (ii) The intrinsic frequency of the F-P sensor can be in the frequency range of $100-170 \mathrm{kHz}$, and resonance oscillation of sensors will occur when PD AE is detected. (iii) The intrinsic frequency design will be more sensitive than the flat response frequency range design.

\section{Data Availability}

The data used to support the findings of this study are currently under embargo while the research findings are commercialized. Requests for data,12 months after publication of this article, will be considered by the corresponding author.

\section{Conflicts of Interest}

The authors declare that there is no conflict of interest regarding the publication of this paper.

\section{Acknowledgments}

We would like to thank Editage (https://www.editage.cn) for English language editing. This research is funded by the Project Supported by the National Natural Science Foundation of China (No. 51607049), and the Fundamental Research Foundation for Universities of Heilongjiang Province (No. LGYC2018JC29).

\section{References}

[1] Y. B. Wang, Y. H. Fan, S. R. Qin et al., "Partial discharge localisation methodology for power transformers based on improved acoustic propagation route search algorithm," IET Science, Measurement \& Technology, vol. 12, no. 8, pp. 10231030, 2018.

[2] M. R. Islam, M. M. Ali, M. H. Lai, K. S. Lim, and H. Ahmad, "Chronology of Fabry-Perot interferometer fiber-optic sensors and their applications: a review," Sensors, vol. 14, no. 4, pp. 7451-7488, 2014.

[3] J. Hwang, S. Seon, and C. S. Park, "Position estimation of sound source using three optical Mach-Zehnder acoustic sensor array," Current Optics and Photonics, vol. 1, no. 6, pp. 573-578, 2017.

[4] L. Liu, P. Lu, H. Liao et al., "Fiber-optic Michelson interferometric acoustic sensor based on a PP/PET diaphragm," IEEE Sensors Journal, vol. 16, no. 9, pp. 3054-3058, 2016.

[5] M. J. F. Digonnet, M. Bishop, and G. S. Kino, "Modeling and measurement of the acoustic lead sensitivity in Sagnac fiber sensor arrays," Journal of Lightwave Technology, vol. 24, no. 7, pp. 2877-2888, 2006.

[6] J. Deng, H. Xiao, W. Huo et al., "Optical fiber sensor-based detection of partial discharges in power transformers," Optics \& Laser Technology, vol. 33, no. 5, pp. 305-311, 2001. 
[7] X. Wang, B. Li, Z. Xiao et al., "An ultra-sensitive optical MEMS sensor for partial discharge detection," Journal of Micromechanics and Microengineering, vol. 15, no. 3, pp. 521-527, 2005.

[8] B. Liu, J. Lin, J. Wang, C. Ye, and P. Jin, "MEMS-based highsensitivity Fabry-Perot acoustic sensor with a $45^{\circ}$ angled fiber," IEEE Photonics Technology Letters, vol. 28, no. 5, pp. 581-584, 2016.

[9] O. C. Akkaya, O. Akkaya, M. J. F. Digonnet, G. S. Kino, and O. Solgaard, "Modeling and demonstration of thermally stable high-sensitivity reproducible acoustic sensors," Journal of Microelectromechanical Systems, vol. 21, no. 6, pp. 13471356, 2012.

[10] W. Zhang, F. Chen, W. Ma, Q. Rong, X. Qiao, and R. Wang, "Ultrasonic imaging of seismic physical models using a fringe visibility enhanced fiber-optic Fabry-Perot interferometric sensor," Optics Express, vol. 26, no. 8, pp. 11025-11033, 2018.

[11] Z. Yu, H. Dai, M. Zhang et al., "High stability and low harmonic distortion PGC demodulation technique for interferometric optical fiber sensors," Optics \& Laser Technology, vol. 109, pp. 8-13, 2019.

[12] American National Standard, "IEEE guide for the detection and location of acoustic emissions from partial discharges in oil-immersed power transformers and reactors," in IEEE Power Engineering Society. IEEE Std C57. 127-2007, pp. 918, Transformers Committee, New York, USA, 2007.

[13] B. Yu, D. W. Kim, J. Deng, H. Xiao, and A. Wang, "Fiber Fabry-Perot sensors for detection of partial discharges in power transformers," Applied Optics, vol. 42, no. 16, pp. 3241-3250, 2003.

[14] L. E. Lundgaard, "Partial discharge. XIII. Acoustic partial discharge detection-fundamental considerations," IEEE Electrical Insulation Magazine, vol. 8, no. 4, pp. 25-31, 1992.

[15] T. Boczar, "Identification of a specific type of PD from acoustic emission frequency spectra," IEEE Transactions on Dielectrics and Electrical Insulation, vol. 8, no. 4, pp. 598-606, 2001.

[16] M. MacAlpine, Z. Zhiqiang, and M. S. Demokan, "Development of a fibre-optic sensor for partial discharges in oil-filled power transformers," Electric Power Systems Research, vol. 63, no. 1, pp. 27-36, 2002.

[17] B. Dong, M. Han, and A. Wang, "Two-wavelength quadrature multipoint detection of partial discharge in power transformers using fiber Fabry-Perot acoustic sensors," in Proceedings Volume 8370, Fiber Optic Sensors and Applications IX, p. 14, Baltimore, MA, USA, 2012. 


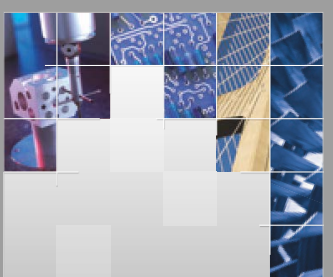

\section{Enfincering}
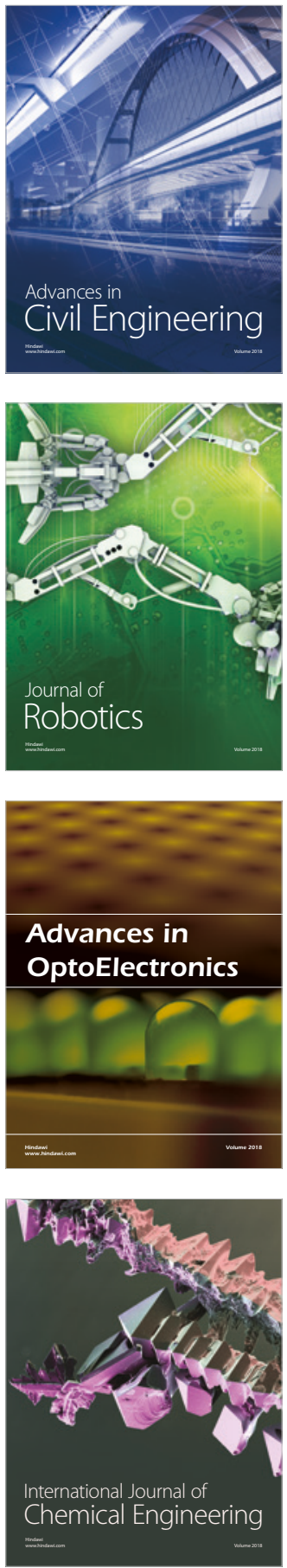

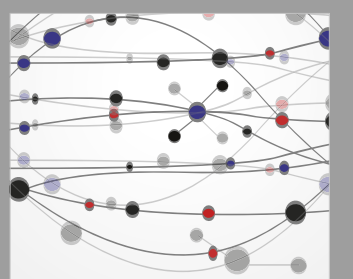

\section{Rotating \\ Machinery}

The Scientific World Journal

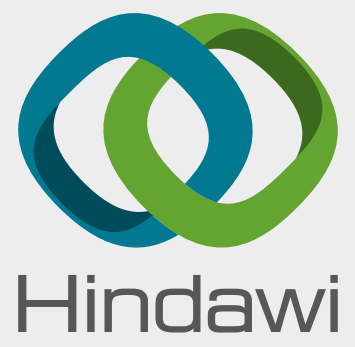

Submit your manuscripts at

www.hindawi.com
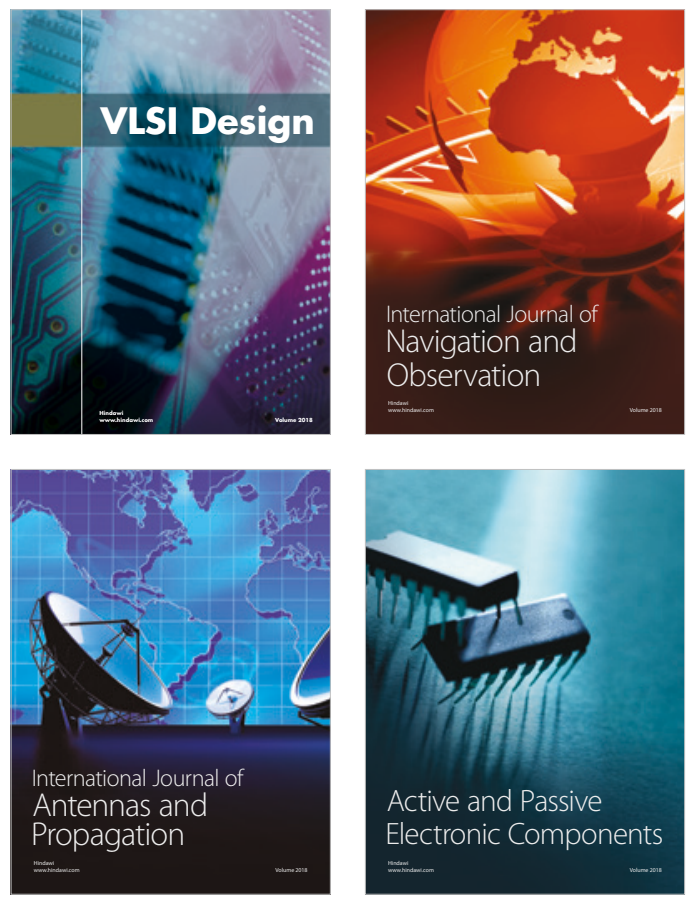
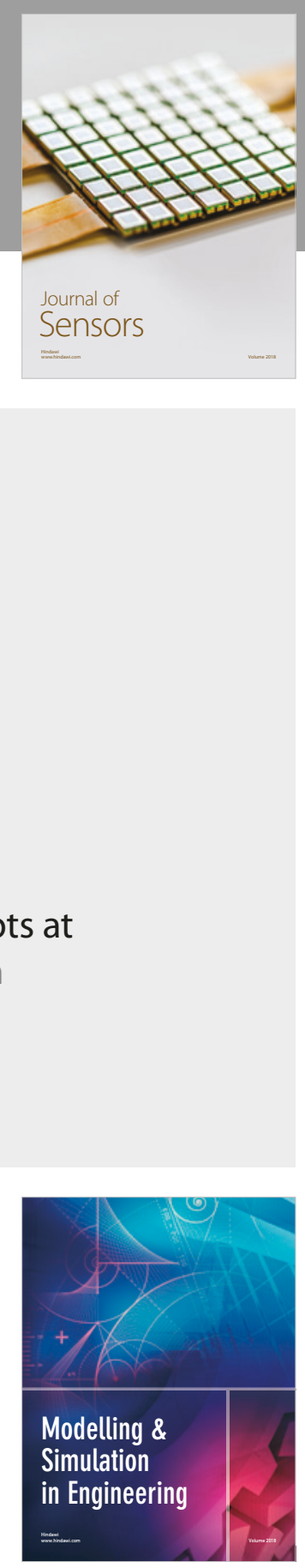

\section{Advances \\ Multimedia}
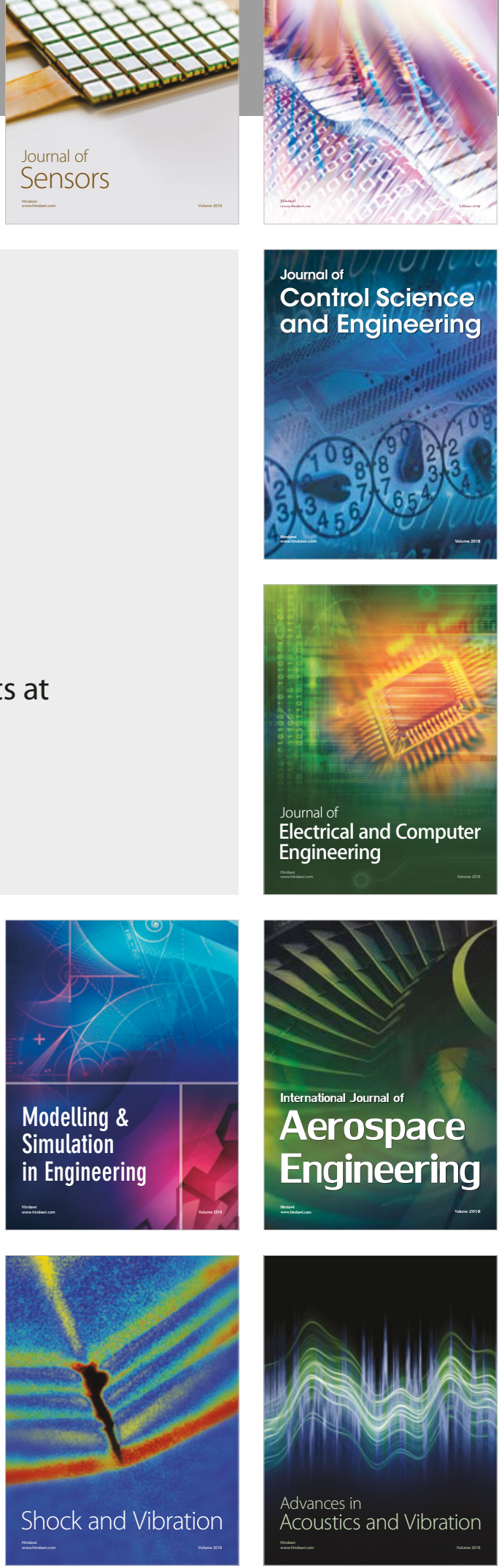